1 Universidade Federal da Paraíba (UFPB) - João Pessoa (PB), Brasil.

camilaribeiroufpb@hotmail. com

2 Universidade Federal da Paraíba (UFPB) - João Pessoa (PB), Brasil. tatiene@de.ufpb.br

3 Universidade Federal da Paraíba (UFPB) - João Pessoa (PB), Brasil. calilunalima@gmail.com

4 Universidade Federal da Paraíba (UFPB) - João Pessoa (PB), Brasil.

luciano_bsfilho@hotmail.

com

\section{Fatores associados à eficiência na Atenção Básica em saúde, nos municípios brasileiros}

Factors associated with efficiency in Primary Health Care, in brazilian municipalities

Camila Ribeiro da Silva1, Tatiene Correia de Souza², Caliandra Maria Bezerra Luna Lima³, Luciano Belas e Silva Filho 4

DOI: 10.1590/0103-1104201811703

RESUMO Este artigo tem por objetivo identificar os fatores que influenciam a eficiência na aplicação dos recursos destinados à Atenção Básica nos municípios brasileiros. Para isso, utilizou-se o modelo de regressão beta inflacionado, uma vez que a variável dependente apresenta valores no intervalo $(0,1]$. De acordo com os resultados, o índice Firjan de desenvolvimento municipal na saúde e serviços de saneamento básico são fatores que influenciam positivamente a eficiência média na Atenção Básica de um município. Por outro lado, o gasto per capita de um município, em Atenção Básica, exerce efeito negativo na eficiência média.

PALAVRAS-CHAVE Atenção Primária à Saúde. Eficiência. Modelos estatísticos.

ABSTRACT This article aims to identify the factors that influence the efficiency in the application of resources destined to Primary Health Care in brazilian municipalities. To this end, the inflated beta regression model was used, since the dependent variable presents values in the interval $(0,1]$. According to the results, the Firjan Index of Municipal Development in health and basic sanitation services are factors that positively influence the average efficiency in the Primary Health Care of a municipality. On the other hand, the per capita expenditure of a municipality, in primary care, exerts a negative effect on the average efficiency.

KEYWORDS Primary Health Care. Efficiency. Statistical models. 


\section{Introdução}

A eficiência em saúde é compreendida como a relação entre o custo e o impacto dos serviços sobre a saúde da população, mantendo-se um nível de qualidade determinado'. A eficiência em políticas públicas traz como referência o montante de recursos envolvidos, com o objetivo de mensurar a otimização ou o desperdício de insumos despendidos no alcance das metas, no sentido de reestruturar a ação para obter, com menor custo e esforço, melhores resultados ${ }^{2}$.

A avaliação do desempenho municipal é uma forma de mensurar a situação dos serviços de saúde no âmbito da Atenção Básica (AB). No Brasil, alguns estudos avaliaram a eficiência na aplicação de recursos públicos municipais e buscaram identificar os impactos sobre os indicadores de atenção à saúde ${ }^{\mathbf{3} 4}$.

Em estudo divulgado pelo jornal folha de São Paulo 5 , em parceria com o Datafolha, foram apresentados resultados de eficiência para 5.281 municípios brasileiros, nos aspectos de saúde, educação e saneamento básico. De acordo com os resultados, foi revelado que apenas $24 \%$ desses municípios foram considerados eficientes, segundo critério adotado no estudo, que levou em conta o valor de eficiência superior a 0,5. Com relação ao indicador de saúde, verificaram-se grandes discrepâncias entre alguns municípios. Por exemplo, os municípios de Cachoeira da Prata (MG), Presidente Lucena (RS) e Altaneira (CE) apresentaram indicadores em saúde de 0,687, 0,786 e 0,586, respectivamente, e foram considerados eficientes de acordo com o ranking.

Por outro lado, os municípios de Boa Viagem (CE) e Campo Alegre (AL) registraram indicadores de saúde bem inferiores quando comparados aos municípios supracitados, sendo de 0,283 e 0,387 , respectivamente, e foram classificados como ineficientes. Apesar dos resultados relevantes do respectivo estudo, vale ressaltar que a eficiência mensurada se refere a três aspectos distintos: saúde, educação e saneamento básico, e que os indicadores de eficiência são dados de forma geral ${ }^{5}$.

Desse modo, torna-se relevante avaliar os fatores que podem influenciar a eficiência na aplicação de recursos em diversos níveis da saúde, sobretudo na $A B$, visto que um sistema eficiente em cuidados primários proporciona melhorias substanciais de saúde, a custos relativamente pequenos, principalmente quando comparados aos dos níveis secundário e terciário ${ }^{6,7}$. É possível a comprovação, através de ampla literatura, dos inúmeros benefícios que um sistema de $\mathrm{AB}$ pode proporcionar, estando bem estruturado e interligado aos outros níveis de atenção, que vão desde o menor uso de serviços de urgência e emergência à melhora na detecção de fatores adversos a intervenções médicas ${ }^{8}$.

Sendo assim, tendo em vista a relevância da aplicação dos recursos de forma eficiente, buscando reduzir gastos desnecessários, o presente artigo justifica-se pela importância de identificar fatores determinantes que possam influenciar a eficiência no gerenciamento de recursos destinados à AB. Para tanto, utilizou-se um modelo de regressão beta inflacionado, a fim de modelar o escore de eficiência na $A B$, uma vez que a variável dependente possui valores no intervalo $(0,1]^{\text {? }}$.

\section{Material e métodos}

Trata-se de um estudo descritivo, de abordagem quantitativa, baseado em dados secundários que foram disponibilizados por Sousa $^{10}$. A autora buscou mensurar escores de eficiência na aplicação de recursos destinados à $\mathrm{AB}$ dos municípios brasileiros, utilizando a metodologia Data Envelopment Analysis (DEA) 11, tendo como base o ano de 2007. A metodologia DEA fundamenta-se nas definições de eficiência técnica proposta por Farrel12. Trata-se de uma técnica não paramétrica para a avaliação da eficiência relativa de um conjunto de Decision Making Units (DMU) homogêneas. Utilizando-se da 
quantidade de recursos consumidos e produtos gerados por unidade, e, com o auxílio da técnica de programação linear, a DEA baseada no melhor desempenho observado constrói a fronteira de eficiência da produção, a qual servirá para a avaliação da eficiência das demais unidades observadas ${ }^{13}$. Na prática, existem outras metodologias usadas na avaliação da eficiência em saúde, a exemplo de fronteira estocástica. No entanto, é possível verificar, através da literatura, a ampla utilidade da técnica DEA na mensuração de eficiência em saúde. Com relação à $\mathrm{AB}$, destacam-se os trabalhos de Bryce et al. ${ }^{14}$, Zavras et al. ${ }^{15} \mathrm{e}$ Rosenman e Friesner ${ }^{16}$.

A amostra foi composta por 3.409 municípios brasileiros, distribuídos nas cinco regiões geográficas, a saber: 284 municípios da região Norte; 1.259 da região Nordeste; 204 da região Centro-Oeste; 720 da região Sul; e 942 da região Sudeste.

Para cada município observado, foi atribuído um valor, que representa a eficiência técnica do município quanto à aplicação dos recursos destinados à $\mathrm{AB}$. As medidas de eficiência na $\mathrm{AB}$ obtidas através da técnica não paramétrica DEA assumem valores no intervalo $(0,1]$. Para fins dessa análise, os municípios que obtiveram valor de eficiência na $\mathrm{AB}$ igual a 1 são denominados plenamente eficientes na aplicação desses recursos.

A base de dados disponibilizada também contém informações de variáveis socioeconômicas e relacionadas à saúde, que foram coletadas em fontes de domínio público, como o Instituto Brasileiro de Geografia e Estatística (IBGE), o Departamento de Informática do Sistema Único de Saúde (Datasus), o Sistema de Informação da Atenção Básica (Siab), o Cadastro Nacional de Estabelecimentos de Saúde (CNES) e a Federação das Indústrias do Estado do Rio de Janeiro (Firjan).

O Índice Firjan de Desenvolvimento Municipal (IFDM) é um estudo realizado pelo sistema Firjan com o propósito de acompanhar anualmente o desenvolvimento socioeconômico dos municípios brasileiros, em três áreas de atuação: Emprego e renda; Educação; e Saúde. Desenvolvido em 2008, o índice se baseia, exclusivamente, em estatísticas públicas oficiais disponibilizadas pelos ministérios do Trabalho, da Educação e da Saúde17.

$O$ índice varia de 0 (mínimo) a 1 (máximo). Quanto mais próximo de 1, maior o nível de desenvolvimento do município em saúde, renda ou educação. Na construção dos índices são utilizadas variáveis relacionadas ao trabalho, à educação e à saúde, como por exemplo, o número de empregos com carteira assinada no município, o número de matrículas na educação infantil e o número de consultas pré-natais realizadas, respectivamente ${ }^{17}$.

A análise estatística foi realizada utilizando medidas descritivas e análise de regressão. A análise descritiva teve por objetivo verificar particularidades entre os municípios observados, com base nos valores médio, mínimo e máximo de algumas variáveis. A análise de regressão foi realizada considerando a classe de modelos de regressão beta inflacionados ${ }^{18}$. Essa classe de modelos é adequada para modelar variáveis que pertencem ao intervalo unitário padrão $(0,1)$, assumindo, inclusive, os valores 0 ou 1 . Maiores detalhes sobre o modelo de regressão beta inflacionado podem ser encontrados em Pereira e Cribari-Neto ${ }^{19}$.

A descrição das variáveis utilizadas no artigo é apresentada no quadro 1. A variável Eficiência na $A B$ (Efic), que assume valores no intervalo $(0,1]$, foi considerada como variável dependente, enquanto as demais variáveis foram utilizadas como explicativas. As variáveis NO, NE, CO e SE são variáveis dicotômicas que foram introduzidas com o objetivo de verificar a influência de cada região geográfica. Para isso, foram considerados os municípios da região Sul como categoria de referência. O nível de significância considerado foi de $5 \%$ (p-valor $<0,05)$. Os dados foram analisados por meio do software estatístico R20. 


\begin{tabular}{|c|c|}
\hline Variável & Definição \\
\hline EFIC & Escores de eficiência para Atenção Básica - ano 2007. \\
\hline NO & Dummy: igual a 1 se o município pertence à região Norte; 0, caso contrário. \\
\hline NE & Dummy: igual a 1 se o município pertence à região Nordeste; 0 , caso contrário. \\
\hline SE & Dummy: igual a 1 se o município pertence à região Sudeste; 0, caso contrário. \\
\hline $\mathrm{CO}$ & Dummy: igual a 1 se o município pertence à região Centro-Oeste; 0 , caso contrário. \\
\hline $\mathrm{PCl}$ & Dummy: igual a 1 se o município participa de consórcio intermunicipal; 0, caso contrário. \\
\hline PODER & Dummy: igual a 1 se o conselho municipal tem poder de decisão; 0 , caso contrário. \\
\hline POP & População do município. \\
\hline MED & Média de médicos por estabelecimento da Atenção Básica. \\
\hline CONSUL & Número de consultas por estabelecimento. \\
\hline IFDMS & Índice Firjan de desenvolvimento municipal na saúde. \\
\hline MED-ENF & Relação entre o número de médicos e enfermeiros da Atenção Básica municipal. \\
\hline SANEA & $\begin{array}{l}\text { Média dos percentuais de famílias atendidas pelas equipes de saúde da família que possuem } \\
\text { água em rede, esgoto e coleta de lixo. }\end{array}$ \\
\hline HOSP & Dummy: igual a 1 se houver hospital no município; 0, caso contrário. \\
\hline IDOS & Proporção da população municipal com idade acima de 60 anos. \\
\hline REG & Dummy: igual a 1 se o município pertence a uma região metropolitana; 0, caso contrário \\
\hline CAP & Dummy: igual a 1 se o município for a capital do estado; 0, caso contrário. \\
\hline GASTO & Gasto per capita municipal em Atenção Básica - ano 2007. \\
\hline
\end{tabular}

Fonte: Elaboração própria.

\section{Resultados e discussão}

\section{Análise descritiva}

De acordo com a análise descritiva, pode-se destacar que o escore de eficiência médio foi 0,72, com valor mínimo de 0,159 para o município de Rio Preto (MG). Do total de municípios da amostra (3.409), apenas 15,26\% se configuram como plenamente eficientes no gerenciamento de recursos destinados à $\mathrm{AB}$. Os estados de Minas Gerais e São Paulo foram os que apresentaram o maior número de municípios com plena eficiência sendo 62 e 61, respectivamente. Na região Nordeste, destacaram-se os estados de Pernambuco, com 33 municípios, e Ceará e Bahia, com 37 e 45, respectivamente. Os melhores índices Firjan de desenvolvimento municipal em renda e em educação foram observados nos municípios de Itabira (MG) e Santa Rita d'Oeste (SP), com valores de 0,997 e 0,956, respectivamente.

Na saúde, os melhores índices Firjan de desenvolvimento municipal foram observados nos municípios de Flórida (PR), Alto Alegre (RS), Rondinha (RS) e Saldanha Marinho (RS), com valores iguais a 1. Por outro lado, o município de São Félix do Tocantins (TO) apresentou o menor IFDM na renda $(0,041)$; Wenceslau Guimarães (BA), o menor índice IFDM na educação $(0,297)$; e São João do Piauí (PI), na saúde $(0,361)$. O maior gasto per capita em $\mathrm{AB}$ foi realizado pelo município de Madre de Deus (BA), cujo valor foi de $\mathrm{R} \$ 1.080$, sendo superior à média do gasto per capita nos demais municípios ( $\mathrm{R} \$ 141,80)$. 
A variável relação entre o número de médicos e enfermeiros na $\mathrm{AB}$ (MED-ENF) é muito importante, tendo em vista que a eficácia dos serviços está relacionada com as categorias profissionais dispostas nos estabelecimentos de saúde. O valor médio para essa variável foi de 1,21, indicando maior número de médicos em relação aos profissionais de enfermagem. Essa relação foi superior a 1 principalmente nas regiões Sudeste (722), Nordeste (603) e Sul (468).

Quanto aos estados, a maior ocorrência desse tipo de situação foi verificada na Bahia (141), em Minas Gerais (447), em São Paulo (176), no Paraná (164), em Santa Catarina
(115) e no Rio Grande do Sul (189). Além disso, quanto às variáveis IDOS e INFANT (quadro 1), verifica-se que os municípios com maior proporção de idosos encontram-se nas regiões sul e sudeste, enquanto a população com menos de 5 anos de idade se destaca nas regiões Norte e Nordeste.

A figura 1 apresenta o histograma e box-plot da EFIC na AB. Nota-se que a distribuição é assimétrica à direita, com grande frequência de pontos no valor igual a 1 . Além disso, a partir do box-plot nota-se que a mediana está mais próxima do primeiro quartil e que não foram destacadas observações discrepantes, ou seja, pontos que excedessem os limites do box-plot.

Figura 1. Histograma e box-plot da variável eficiência na Atenção Básica.

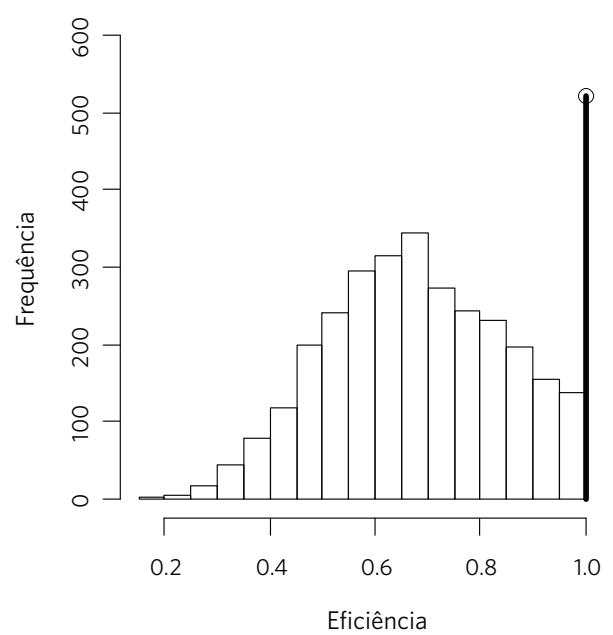

Fonte: Elaboração própria

A variável gasto per capita em AB (GASTO) pode ser considerada como um fator muito importante no âmbito da gestão municipal, tendo em vista que possibilita a observação do montante de recursos que cada ente administrativo disponibiliza para a $\mathrm{AB}$, por habitante. Neste sentido, a tabela 1 apresenta o número de municípios por região para diferentes faixas de gasto per capita em AB. Observa-se

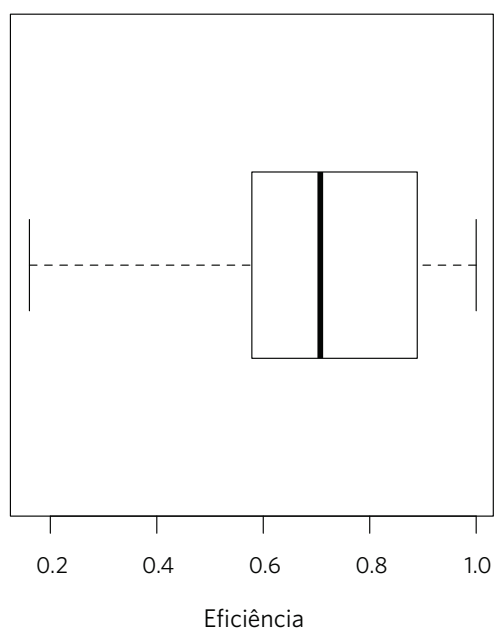

que a maioria dos municípios possui GASTO entre $\mathrm{R} \$ 100$ e $\mathrm{R} \$ 499$. O maior número de municípios com gasto per capita superior a $\mathrm{R} \$ 500$ encontra-se nas regiões Sul e Sudeste, totalizando 35 municípios, ao passo que os com valor per capita acima de $\mathrm{R} \$ 800$, situam-se na região Nordeste, no município de Madre de Deus (BA), e na região Sudeste, em Carapebus (RJ). 
Tabela 1. Faixa de gasto per capita em Atenção Básica, segundo número de municípios por região

\begin{tabular}{lrrrrr}
\hline Gasto per capita & \multicolumn{5}{c}{ Região } \\
\cline { 2 - 6 } em $A B$ & Centro-Oeste & Nordeste & Norte & Sudeste & Sul \\
\hline Acima de $R \$ 100$ & 123 & 627 & 93 & 556 & 538 \\
Acima de $R \$ 500$ & 5 & 2 & 2 & 18 & 17 \\
Acima de $R \$ 800$ & 0 & 1 & 0 & 1 & 0 \\
\hline Total & 128 & 630 & 95 & 575 & 555 \\
\hline
\end{tabular}

Fonte: Elaboração própria.

\section{Ajuste do modelo de regressão beta inflacionado}

A tabela 2 apresenta os resultados do modelo de regressão beta inflacionado para a modelagem dos escores de eficiência, identificando os fatores que influenciam a eficiência média na $\mathrm{AB}$ dos municípios. Todas as variáveis apresentam significância ao nível de 5\% (p-valor $<0,05)$. Conforme tabela 2, para o modelo da média, nota-se que a variável população do município (POP) influencia positivamente a eficiência média na $\mathrm{AB}$, ou seja, os municípios com maior população tendem a apresentar maiores escores de eficiência na $\mathrm{AB}$.

Observa-se, também, que o IFDM na saúde influencia positivamente a eficiência média do município. Isto é, municípios com melhores níveis de desenvolvimento em saúde tendem a apresentar maiores escores de eficiência na AB. A variável SANEA, que representa o percentual médio de famílias com acesso aos serviços essenciais de saneamento básico, também influencia de forma positiva a eficiência média do município. Ou seja, a população que convive com serviços de saneamento básico adequados apresenta menores riscos de ser acometida por algum tipo de doença $\mathbf{a}^{21}$.

Segundo Gonzalez, Tozoni-Reis e Diniz²1, os transtornos oriundos do acúmulo de lixo em locais inadequados, além de contribuir para o aparecimento de doenças, faz com que a comunidade encontre saídas próprias para a resolução deste problema - uma delas é a promoção de queimadas do lixo, que trazem, como consequências, a produção de fumaças tóxicas e o risco de incêndios, ambos prejudiciais à saúde.

Esse achado corrobora e evidencia o conceito ampliado de saúde, formulado durante a VIII Conferência Nacional de Saúde, no qual a saúde é entendida como:

resultante das condições de alimentação, habitação, educação, renda, meio ambiente, trabalho, transporte, emprego, lazer, liberdade, acesso e posse da terra e acesso aos serviços desaúde. Sendo assim, é principalmente resultado das formas de organizaçãosocial, de produção, as quais podem gerar grandes desigualdades nos níveis de vida22(382).

Por outro lado, verifica-se que a variável GASTO exerce efeito negativo na eficiência média, isto é, municípios com maiores gastos per capita tendem a apresentar menor eficiência média na aplicação de recursos destinados à AB. Essa constatação pode ser explicada pelo tipo de cuidado que deve ser prioritariamente empregado na $A B$. Tais práticas devem perpassar as tecnologias leves do cuidado, com o objetivo de dar resolutividade às demandas e necessidades de saúde dos sujeitos em seus territórios ${ }^{23}$.

Coelho e Jorge ${ }^{24}$ apresentam o acolhimento, o vínculo e a atenção integral como tecnologias leves no trabalho em saúde, as quais Mehry e Franco ${ }^{23}$ afirmam estarem centradas no campo das relações, evidenciando o diálogo e o envolvimento entre profissionais e usuários. 
Em relação às variáveis $\mathrm{NO}, \mathrm{NE}, \mathrm{CO}$, respectivamente ligadas às regiões Norte, Nordeste e Centro-Oeste, é possível concluir que os municípios nelas localizados apresentam maior eficiência média na aplicação dos recursos destinados à $\mathrm{AB}$, em relação aos municípios da região Sul. Conforme Sousa ${ }^{10}$, esse resultado pode ser explicado pelo fato de que os bons escores na região Nordeste são reflexos de relações mais altas entre produtos e insumos, e não necessariamente melhores prestações de serviços.

É provável que a qualidade dos serviços de $\mathrm{AB}$ seja melhor nos municípios da região Sul. Entretanto, se nesses municípios a produção de tais serviços estiver relacionada a custos proporcionalmente maiores, essas localidades não poderão ser consideradas como exemplos de melhores práticas, isto é, como tendo alguns dos melhores desempenhos ${ }^{10}$. Além disto, tal conclusão coincide com o apresentado por Dias ${ }^{25}$, que também observou menores escores de eficiência nas regiões Sul e Sudeste, justificados também pelas melhores relações de insumo e produto.

Quanto ao modelo que se refere à probabilidade de o município ser considerado plenamente eficiente $(\alpha)$, destacam-se como significativas as variáveis PCI, CONSUL, CAP e SE, além das variáveis GASTO, POP, IDOS e NE, já citadas (quadro 1). Nota-se que municípios com maiores populações, maior número de consultas por estabelecimento de saúde, e que pertencem às regiões Nordeste ou Sudeste, apresentam maiores chances de serem plenos no gerenciamento de recursos destinados à $\mathrm{AB}$.

Em contrapartida, municípios que participam de consórcio intermunicipal, apresentam maiores gastos per capita em $\mathrm{AB}$, possuem maior proporção da população acima de 60 anos e são capitais apresentam menores chances de serem plenamente eficientes no gerenciamento de recursos destinados à $\mathrm{AB}$. Tais achados costumam ser explicados pelo fato de que, por exemplo, a faixa etária da população acima de 60 anos tende a apresentar maior incidência de doenças crônicas, necessitando de atendimento contínuo, por sua vez, gerando maiores despesas. Isto faz com que sejam efetuados maiores gastos em saúde, ocasionando menores escores de eficiência ${ }^{10}$.

Além disso, para que haja uma eficiente aplicação dos recursos disponíveis, é primordial que o município apresente uma excelente gestão administrativa, que disponha de conhecimento a respeito do assunto e que não busque apenas aplicar o recurso, mas entenda de que forma ele será útil em cada departamento. $\mathrm{O}$ fato de as capitais apresentarem menor chance de serem plenamente eficientes pode ser reflexo de alguns fatores, a saber: a dificuldade na aplicação dos recursos, a diversidade de acometimentos em saúde ou a demanda de um contingente populacional que, geralmente, é maior do que a dos outros municípios.

Com relação ao modelo para a precisão, apenas as variáveis MED-ENF, NE e SE (quadro 1) foram significativas ao nível de $5 \%$ (p-valor $<0,05$ ). Este resultado indica que os municípios com maior número de médicos em relação ao de enfermeiros na $\mathrm{AB}$ tendem a apresentar escores de eficiência menos dispersos, assim como nos municípios localizados nas regiões Nordeste e Sudeste. 
Tabela 2. Modelo de regressão beta inflacionado para o escore de eficiência na Atenção Básica à saúde, para os municípios brasileiros

\begin{tabular}{|c|c|c|c|}
\hline \multicolumn{4}{|c|}{ Modelo para a média $(\mu)$} \\
\hline Variável & Estimativa & Erro-padrão & $\mathrm{p}$-valor \\
\hline Constante & 0,2645 & 0,1258 & 0,0355 \\
\hline POP & $2,050 \times 10-6$ & $3,097 \times 10-7$ & $<0,001$ \\
\hline IFDMS & 0,5470 & 0,1482 & $<0,001$ \\
\hline GASTO & $-0,0015$ & 0,0001 & $<0,001$ \\
\hline N & 0,2538 & 0,0646 & $<0,001$ \\
\hline NE & 0,3776 & 0,0413 & $<0,001$ \\
\hline $\mathrm{CO}$ & 0,1616 & 0,0649 & 0,0128 \\
\hline SANEA & 0,1917 & 0,0677 & 0,0047 \\
\hline \multicolumn{4}{|c|}{ Modelo para a massa de probabilidade em um $(\alpha)$} \\
\hline Constante & $-0,7092$ & 0,2148 & $<0,001$ \\
\hline GASTO & $-0,0055$ & 0,0006 & $<0,001$ \\
\hline POP & $3,374 \times 10-6$ & $6,567 \times 10-7$ & $<0,001$ \\
\hline IDOS & $-7,6010$ & 1,771 & $<0,001$ \\
\hline $\mathrm{PCl}$ & $-0,4685$ & 0,1243 & 0,0002 \\
\hline CONSUL & $4,499 \times 10-5$ & $1,084 \times 10-5$ & $<0,001$ \\
\hline CAP & $-1,976$ & 0,8841 & 0,0255 \\
\hline NE & 0,3045 & 0,1249 & 0,0145 \\
\hline SE & 0,3539 & 0,1440 & 0,0141 \\
\hline \multicolumn{4}{|c|}{ Modelo para a precisão $(\phi)$} \\
\hline Constante & 1,6647 & 0,0701 & $<0,001$ \\
\hline MED-ENF & 0,1914 & 0,0626 & 0,0023 \\
\hline NE & 0,1424 & 0,0590 & 0,0159 \\
\hline SE & 0,1179 & 0,0472 & 0,0125 \\
\hline
\end{tabular}

Fonte: Elaboração própria.

\section{Considerações finais}

Diante da importância dos fatores que podem afetar a eficiência na aplicação de recursos destinados à $\mathrm{AB}$, o presente estudo colabora para o conhecimento destes, além de avaliar em que direção eles se relacionam com a eficiência na $\mathrm{AB}$. Os resultados demonstrados podem subsidiar ações de políticas públicas na área de planejamento e gestão administrativa, com o propósito de buscar a melhor aplicabilidade dos recursos disponibilizados nesse nível de atenção.
Pelo exposto, observa-se que maiores gastos per capita em $\mathrm{AB}$, não necessariamente representam maior eficiência na aplicação dos recursos. Isto pode ser aplicado, por exemplo, em municípios menores, que despendem menos recursos e buscam atender à demanda de serviços com o que dispõem, fazendo com que apresentem uma postura diferenciada e atinjam melhores relações de custo-benefício.

Ademais, verifica-se a relevância dos serviços de saneamento básico que são oferecidos à população, uma vez que estes se configuram 
como importantes determinantes sociais da saúde, refletindo, inclusive, na eficiência em $\mathrm{AB}$ do município. O IFDM na saúde também se mostra como um fator importante no que diz respeito à eficiência na $\mathrm{AB}$ do município. Em suma, bons índices de desenvolvimento municipal na saúde e saneamento básico de qualidade são pontos cruciais para a busca de uma maior eficiência média na aplicação de recursos na $\mathrm{AB}$, pelo gestor.

Sabendo que, no Brasil, os recursos destinados aos estados e municípios para o provimento de seus serviços são limitados, inclusive, aqueles reservados para aspectos essenciais, como é o caso da saúde, ressalta-se a importância da aplicação dos recursos de forma eficiente, na tentativa de despender menos custos e evidenciar um modelo de gestão eficaz. Desse modo, poderão ser alcançados resultados satisfatórios, investindo e gerindo os recursos de forma consciente, evitando gastos desnecessários.

Neste sentido, o presente artigo visa trazer uma contribuição no que diz respeito ao gerenciamento de recursos destinados à $\mathrm{AB}$, proporcionando um melhor direcionamento à aplicação de recursos, a fim de, assim, poder buscar uma maior eficácia nos serviços prestados à população, no âmbito da gestão municipal.

\section{Referências}

1. Viacava F, Ugá MAD, Porto S et al. Avaliação de Desempenho de Sistemas de Saúde: um modelo de análise. Ciênc. Saúde Colet. [internet]. 2012 abr [acesso em 2018 mar 13]; 17(4):921-934. Disponível em: $\quad<$ http://www.scielo.br/scielo.php?script=sci arttext\&pid=S1413-81232012000400014\&lng=en> .

2. Fagundes H, Moura AB. Avaliação de programas e políticas públicas. Rev. Textos Cont. 2009; 8(1):89103.

3. Varela OS, Martins GDA, Fávero LPL. Desempenho dos municípios paulistas: uma avaliação de eficiência da atenção básica à saúde. Rev. Adm. 2012; 47(4):624-637.

4. Marinho A. Avaliação da eficiência técnica nos serviços de saúde nos municípios do Estado do Rio de Janeiro. Rev. Bras. Economia. 2003; 57(3):515-534.
5. Folha de São Paulo. Ranking inédito revela que só $24 \%$ das cidades são eficientes. São Paulo: Folha de São Paulo; 2016 [acesso em 2018 mar 11]. Disponível em: http://temas.folha.uol.com.br/remf/ranking-de-eficiencia-dos-municipios-folha/ranking-inedito-revela-que-so-24-das-cidades-sao-eficientes. shtml.

6. Atun R. What are the advantages and disadvantages of restructuring a health care system to be more focused on primary care services. Copenhagen: Health Evidence Network; 2004.

7. Engstrom S, Foldevi M, Borquist LS. Is general practice effective: A systematic literature review. Scand. J. Prim. Health Care. 2001;19:131-144.

8. Kravitz R, Duan BJ. Evidence-based medicine, heterogeneity of treatment effects, and the trouble with averages. Milbank Q. 2004; 82(4):661-687. 
9. Ospina R, Ferrari SLP. On Bias Correction in a Class of Inflated Beta Regression Models. Intern. J. Statistics Probab. 2012; 1(2):1-14.

10. Sousa MCS. Mensurando a eficiência da atenção primária à saúde em presença de outliers e fatores exógenos: uma análise em dois estágios [Trabalho apresentado à banca examinadora como parte dos requisitos do Concurso de Professor Titular da Universidade Federal da Paraíba]. [Brasília, DF]: 2011.

11. Charnes A, Cooper WW, Rhodes E. Measuring the efficiency of decision making units. Eur. J. Oper. Res. 1978; 2:429-444.

12. Farrel, MJ. The Mensurement of Productive Efficiency. J. R. Stat. Soc. 1957. 120:253-290.

13. Ligarda L, Naccha M. La eficiencia de las organizaciones de salud a través del análisis envolvente de datos. Microrredes de La Dirección de Salud IV Lima Este 2003. An. Fac. Med. 2006; 67(2):142-151.

14. Bryce CL, Engberg JB, Wholey DR. Comparing the agreement among alternative models in evaluating HMO efficiency. Health Serv. Res. 2000; 35(2):509528.

15. Zavras AI, Tsakos G, Economou C, et al. Using DEA to evaluate efficiency and formulate policy with in a greek national primary health care network. J. Med. Syst. 2002; 26:285-292.

16. Rosenman R, Friesner D. Scope and scalein efficiencies in physician practices. Health Econ. 2004; 13:1091-1116.

17. Federação das Indústrias do Estado do Rio de Janeiro. IDFM 2015: Índice Firjan de Desenvolvimento Municipal. Rio de Janeiro: Firjan; 2015 [acesso em 2018 mar 1]. Disponível em: http://publicacoes. firjan.org.br/ifdm/2015/files/assets/common/downloads/publication.pdf.
18. Ospina R, Ferrari SLP. A general class of zero-or-one inflated beta regression models. Comput. Stat. Data Anal. 2012; 56(6):1609-1623.

19. Pereira TL, Cribari-Neto F. Detecting Model Misspecification in Inflated Beta Regressions. Commun. Stat. Simul. Comput. 2014; 43:631-656.

20. R Core Team. R: A language and Environment for Statistical Computing. Vienna: the R Foudation for Statistical Computing; 2013.

21. Gonzalez LTV, Tozoni-Reis MFC, Diniz RES. Educação ambiental na comunidade: uma proposta de pesquisa-ação. Rev. Eletrônica Mestr. Educ. Ambient. 2007; 18:1-20.

22. Brasil. Ministério da Saúde. VIII Conferência Nacional de Saúde. Brasília, DF: MS; 1986.

23. Merhy EE, Franco TB. Por uma Composição Técnica do Trabalho Centrada nas Tecnologias Leves e no Campo Relacional. Saúde debate. 2003 set-dez; 27(65):316-323.

24. Coelho MO, Jorge MSB. Tecnologia das relações como dispositivo do atendimento humanizado na atenção básica à saúde na perspectiva do acesso, do acolhimento e do vínculo. Ciênc. Saúde Colet. [internet]. 2009 out [acesso em 2018 mar 13]; 14(Suppl1):1523-1531. Disponível em: <http://www. scielo.br/scielo.php?script=sci_arttext\&pid=S1413$-81232009000800026 \& \operatorname{lng}=\mathrm{en}>$.

25. Dias, RH. Eficiência da atenção primária à saúde nos municípios brasileiros [dissertação]. [Brasília, DF]: Universidade de Brasília; 2010.50 p.

Recebido em 11/10/2017

Aprovado em 19/03/2018

Conflito de interesses: inexistente

Suporte financeiro: Coordenação de Aperfeiçoamento de Pessoal de Nível Superior 